\title{
Interfacial fluctuation-dissipation processes and contact angles of thin liquid films
}

\author{
Peter A. Kralchevsky, Ivan B. Ivanov and Antony S. Dimitrov \\ Laboratory of Thermodynamics and Physico-chemical Hydrodynamics, Faculty of Chemistry, University of Sofia, \\ Sofia 1126 , Bulgaria
}

Received 23 May 1991; in final form 9 September 1991

\begin{abstract}
The role of surface viscosity in interfacial fluctuation-dissipation processes is studied. As a result, a new method for calculation of the correlations between interfacial dynamic variables is developed. It is applied to investigate the effect of the suppression of fluctuation capillary waves in thin liquid films on the value of the film contact angle.
\end{abstract}

\section{Introduction}

Since the pioneering work of Mandelstam [1], considerable progress in the study of fluctuation capillary waves has been achieved - reviews of the results for a single interface are given in refs. [2,3], and for thin liquid films in ref. [4]. However, the role of surface viscosity in the interfacial fluctuation-dissipation processes is still not well understood. The recent treatment of the surface stress tensor as an interfacial excess quantity [5] and the hydrodynamic theory of fluctuations [6] are applied (see section 2) to the fluctuation capillary wave problem. In particular, we follow the approach of Landau and Lifshitz [6], according to which the flow in the bulk of the liquid can be treated as the motion of an ideal fluid. As a result, a new simple method for calculating both the correlations between different couples of interfacial variables and the equilibrium mean values is developed. It is applied to calculate the contribution of the surface corrugations to the interfacial free energy (section 3 ). In the case of thin films, this free energy is related to the film contact angle, which is an experimentally measurable quantity. In many cases, the measured contact angles are larger than the values predicted by theories, in which the only source of attraction is the van der Waals force, see the discussion in section 4 . We believe that in some cases this discrepancy between theory and experiment can be removed by taking into account the effect of the surface corrugations which increase the surface area, compared with its value for the macroscopically observable non-disturbed surface. Indeed, the repulsive forces in an equilibrium thin film damp the surface corrugations and thus make the surface dilation (due to corrugations) smaller for the film surface compared with a single surface. This leads to a decrease of the excess surface free energy and to an increase of the contact angle. The relevant equations are derived and discussed in this paper.

\section{Capillary waves and surface stress tensor}

It is assumed in the theory of surface waves [6] that the surface is involved in wave motion, which decays in the depth of the liquid. In the case of fluctuation capillary waves, the wave motion is due to the random Brownian forces acting on the molecules of the interfacial layer. According to the hydrodynamic theory of fluctuations, the random Brownian forces (the random stress tensor) are constitutionally related to the forces of viscous friction $[6,7]$ and, therefore, to the interfacial stress tensor $\tau$. The general component representation of $\tau$ is $[5,8,9]$

$\tau=a_{\alpha} a_{\beta} \tau^{\alpha \beta}+a_{\alpha} n \tau^{\alpha(n)}$. 
(The Greek indices take values $I$ and 2 , and summation over the repeating indices is carried out.) Here, $a_{1}$ and $a_{2}$ are the vectors of the surface basis, and $n$ is the running unit normal to the surface. The components $\tau^{\alpha(n)}$ are called "transverse shear resultants" [10]. The normal projection of the surface linear momentum balance reads

$\tau^{\alpha(n)}{ }_{, \alpha}+b_{\alpha \beta} \tau^{\alpha \beta}-\left(T_{i}^{(n n)}-T_{\mathfrak{l i}}^{(n n)}\right)=0 ;$

see eq. (228) in ref. [5], eq. (3.40) in ref. [9] or eq. (12.13) in ref. [8]; cf. also refs. [11-14] for the mathematical formalism. Here, $b_{\alpha \beta}$ are the components of the surface curvature $\mathbf{b}=-\nabla_{11} n$ with $\nabla_{\mathrm{I}}=a^{\alpha}\left(\partial / \partial u^{\alpha}\right)$ being the surface gradient operator; $T_{l}^{(n n)}$ and $T_{\mathrm{II}}^{(n n)}$ are the normal components of the bulk stress tensor due to the two neighboring fluid phases. Eq. (2.2) is, in fact, a generalization accounting also for the viscous effects of the Laplace equation of capillarity (see e.g. ref. [2]). (The mass of the interface is neglected in eq. (2.2).)

According to Scriven [15] and Eliassen [12], $\tau^{\alpha \beta}$ can be expressed in the form,

$$
\begin{gathered}
\tau^{\alpha \beta}=\sigma a^{\alpha \beta}+\xi_{2} a^{\alpha \beta} a^{\mu \nu} d_{\mu \nu} \\
+\eta_{2}\left(2 d^{\alpha \beta}-a^{\alpha \beta} a^{\mu \nu} d_{\mu \nu}\right),
\end{gathered}
$$

where $a^{\alpha \beta}=a^{\alpha} \cdot a^{\beta}$ is the surface metric tensor, $\sigma$ is the interfacial (surface) tension,

$d^{\mu \nu}=\frac{1}{2}\left(v^{\mu, \nu}+v^{\nu, \mu}-2 b^{\mu \nu} v^{(n)}\right)$

is the surface rate-of-strain tensor,

$\boldsymbol{v}=\boldsymbol{a}_{\mu} v^{\mu}+\boldsymbol{n} v^{(n)}$

is the velocity of a surface point, and $\xi_{2}$ and $\eta_{2}$ are the two-dimensional (surface) dilational and shear viscosities, respectively.

Let us now consider capillary waves of small amplitude $\zeta=\zeta(x, y)$ on a horizontal interface (the plane $x y$ coincides with the undisturbed interface). Then, by using eqs. (2.3) $-(2.5)$, one can prove that

$$
\begin{gathered}
b_{\alpha \beta} \tau^{\alpha \beta}=\sigma \Delta_{2} \zeta+\sigma\left(\zeta^{2}\right), \\
\Delta_{2}=\frac{\partial^{2}}{\partial x^{2}}+\frac{\partial^{2}}{\partial y^{2}} .
\end{gathered}
$$

One sees that the surface dilational and shear viscosities, $\xi_{2}$ and $\eta_{2}$, do not contribute to the linear term on the right-hand side of eq. (2.6).

On the other hand, the components of the surface stress tensor $\tau^{\alpha(n)}$ have predominantly a viscous nature, see ref. [5] p. 114. Hence, by extending Newton's law of viscous friction, one can write

$\tau_{\alpha}^{(n)}=\chi_{2} V_{, \alpha}^{(n)}=\chi_{2} \frac{\partial \zeta_{, \alpha}}{\partial t}$.

(For a more general form of eq. (2.7), see eq. (2.13) below.) Here, $\chi_{2}$ is the coefficient of transverse shear viscosity and the relationship,

$V^{(n)}=\frac{\partial \zeta}{\partial t}$,

has been used. The physical meaning of $\chi_{2}$ is illustrated in fig. 1, where the surface molecules are schematically depicted as small rectangles: the relative vertical displacement of the neighboring surface molecules during the wave motion gives rise to transverse viscous friction.

According to eq. (4.26) in ref. [9], the energy dissipated per unit time due to the effect of the transverse viscous friction is

$\frac{\delta W_{\mathrm{s}}}{\delta t}=\left(\tau^{\alpha(n)} \boldsymbol{a}_{\alpha} \boldsymbol{n}\right):\left(\nabla_{\mathrm{II}} \boldsymbol{V}+U_{\mathrm{II}} \times W\right)$,

where $t$ is the time, $U_{\mathrm{II}}$ is the surface idemfactor and $W$ is the surface angular velocity (one can write $\boldsymbol{W}=\boldsymbol{n} \times(\mathrm{d} \boldsymbol{n} / \mathrm{d} t)$, see ref. [14]; the double scalar product, ":", of two dyadics is defined according to Gibbs - cf, also [14]). In linear approximation with respect to $\zeta$, eq. (2.9) reads

$\frac{\delta W_{\mathrm{s}}}{\delta t}=\tau^{\alpha(n)} V_{, \alpha}^{(n)}$.

Hence, the respective rate of entropy production is

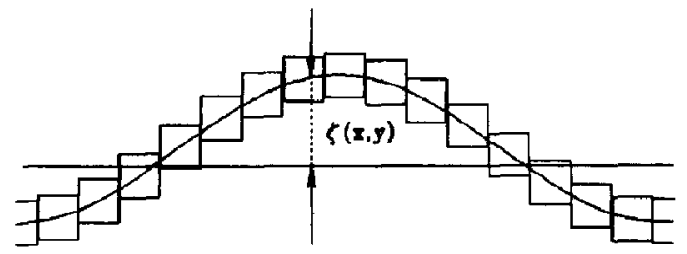

Fig. 1. Sketch of the relative displacement between the surface molecules (the rectangles) during the capillary wave motion. 


$$
\begin{gathered}
\frac{\partial}{\partial t} \int S_{\chi} \mathrm{d} A=\int \frac{1}{T} \tau^{\alpha(n)} V_{, \alpha}^{(n)} \mathrm{d} A \\
=\int \frac{\chi_{2}}{T}\left(V^{(n)}{ }_{, \alpha}\right)^{2} \mathrm{~d} A,
\end{gathered}
$$

where $S_{\chi}$ is the entropy per unit area and $T$ is the temperature; the integration is carried out over the surface, and eqs. (2.7) and (2.10) have also been used. In accordance with the theory of fluctuations in fluid dynamics [6], $\tau$ can be considered as a mean stress tensor and a random surface stress tensor, $s$, can be introduced. The random counterparts of $\tau^{\alpha(n)}$ will be denoted here by $s^{\alpha(n)}$. By following the standard procedure (see ref. [6], chap. XVII, or ref. [7], chap. IX) from eq. (2.11) we derived the following equation for the correlations between the random transverse shear resultants $s^{\alpha(n)}$ :

$$
\begin{aligned}
& \left\langle s^{\alpha(n)}(\boldsymbol{r}, t) s^{\beta(n)}\left(\boldsymbol{r}^{\prime}, t^{\prime}\right)\right\rangle \\
& =2 k_{\mathbf{B}} T \chi_{2} a^{\alpha \beta} \delta\left(\boldsymbol{r}-\boldsymbol{r}^{\prime}\right) \delta\left(t-t^{\prime}\right),
\end{aligned}
$$

where $\boldsymbol{r}$ and $\boldsymbol{r}^{\prime}$ are position vectors of two surface points, $t$ and $t^{\prime}$ are two different time moments, $k_{\mathrm{B}}$ is the Boltzmann constant, and $\delta$ is the Dirac delta function.

Eq. (2.9) suggests the following generalization of the constitutive relation $(2.7)$ :

$\tau_{\alpha}^{(n)}=\chi_{2}\left(V_{, \alpha}^{(n)}+b_{\alpha \beta} V^{\beta}+\varepsilon_{\alpha \beta} W^{\beta}\right)$,

where $\varepsilon_{\alpha \beta}$ is the two-dimensional alternator $[11,12]$. Then, instead of eq. (2.11), one obtains

$$
\begin{aligned}
& \frac{\partial}{\partial t} \int S_{\chi} \mathrm{d} A \\
& =\int \frac{\chi_{2}}{T}\left(V^{(n)}{ }_{, \alpha}+b_{\alpha \beta} V^{\beta}+\varepsilon_{\alpha \beta} W^{\beta}\right)^{2} \mathrm{~d} A .
\end{aligned}
$$

The linear approximations (with respect to $\zeta$ ) of eqs. (2.13) and (2.14) coincide with eqs. (2.7) and (2.11), respectively. It is noteworthy that the more general eq. (2.14) leads to the same fluctuation-dissipation relationship, eq. (2.12), as eq. (2.11).

According to Landau and Lifshitz [6], in the case of surface waves the dissipation of energy takes place mainly in a narrow interfacial zone and can be accounted for by $\tau^{\alpha(n)}$; for the definition of $\tau^{\alpha(n)}$ as surface excess see ref. [5]. Thus, the liquid motion in the bulk phases can be treated as a potential flow of an ideal fluid, obeying the equation $\nabla^{2} \varphi=0$,

where $\varphi$ is the potential of the velocity field, $v=\nabla \varphi$. Then

$T_{\mathrm{I}}^{(n n)}-T_{\mathrm{II}}^{(n)}=-\left(P_{\mathrm{I}}-P_{\mathrm{II}}\right)$,

where $P_{\mathrm{I}}$ and $P_{\mathrm{II}}$ are the pressures in the two neighboring fluids at the interface. By using eqs. (2.6), (2.7) and (2.16), one can represent eq. (2.2) in the form,

$P_{\mathrm{II}}-P_{\mathrm{I}}=\sigma \Delta_{2} \zeta+\chi_{2} \Delta_{2} \frac{\partial \zeta}{\partial t}+s^{\alpha(n)}{ }_{, \alpha}$,

where the random transverse shear resultants $s^{\alpha(n)}$ are also taken into account as required by the theory of hydrodynamic fluctuations [6,7]. It is worthwhile noting that from the three surface viscosity coefficients $\xi_{2}, \eta_{2}$ and $\chi_{2}$, only the last appears in eq. (2.17).

The combination of eqs. (2.12) and (2.17) enables one to solve different problems concerning fluctuation capillary waves. Examples are considered below.

\section{Surface free energy and fluctuation capillary waves}

The increase of the surface area due to surface waves of small amplitude $\zeta(x, y)$ is $[4,6]$

$$
\begin{aligned}
\Delta A & =\frac{1}{2} \iint\left[\left(\frac{\partial \zeta}{\partial x}\right)^{2}+\left(\frac{\partial \zeta}{\partial y}\right)^{2}\right] \mathrm{d} x \mathrm{~d} y \\
& =\frac{1}{2} \int \mid \nabla_{\left.\mathrm{II} \zeta\right|^{2} \mathrm{~d} A .}
\end{aligned}
$$

Then the mean surface dilation per unit area, $\alpha=$ $\Delta A / A$, and the respective capillary wave contribution to the surface free energy, $f_{\mathrm{cw}}$, are

$\alpha=\frac{1}{2 A} \int\left|\nabla_{\mathrm{II}} \zeta\right|^{2} \mathrm{~d} A=\frac{1}{2}\left\langle\left|\nabla_{\mathrm{II}} \zeta\right|^{2}\right\rangle$,

$f_{\mathrm{cw}}=\sigma \alpha=\frac{1}{2} \sigma\left\langle\left|\nabla_{11} \zeta\right|^{2}\right\rangle$.

Our aim below is to calculate, by using the approach developed in the present section, $f_{\mathrm{cw}}$ in three different cases: (i) interface between two semi-infinite fluid phases, (ii) surface of a thin liquid film on solid substratum, and (iii) a free thin liquid film. 


\subsection{Single liquid surface}

For an interface liquid-gas, one can write [6]

$P_{\mathrm{I}}=-\rho g \zeta-\rho \frac{\partial \varphi}{\partial t}, \quad P_{\mathrm{II}}=$ const.,

$\left(\begin{array}{l}\partial \varphi \\ \partial z\end{array}\right)_{z=0}=\frac{\partial \zeta}{\partial t}$,

where $\rho$ is the liquid mass density, $g$ is the acceleration of gravity, the $z$-axis is directed vertically and the plane $z=0$ corresponds to the undisturbed surface. Let us define the Fourier transform of $\zeta$ :

$$
\begin{aligned}
& \zeta(r, t) \equiv \mathscr{F}[\tilde{\zeta}(\boldsymbol{k}, \omega)] \\
& \quad=\int_{-\infty}^{+\infty} \mathrm{d} \omega \int \mathrm{d} k \exp [\mathrm{i}(\boldsymbol{k} \cdot \boldsymbol{r}-\omega t)] \tilde{\zeta}(\boldsymbol{k}, \omega),
\end{aligned}
$$

where both $\boldsymbol{r}$ and $\boldsymbol{k}$ are two-dimensional vectors,

$r=(x, y, 0), \quad k=\left(k_{x}, k_{y}, 0\right)$.

Similarly, one can write

$\boldsymbol{s}^{\alpha(n)}(\boldsymbol{r}, t)=\mathscr{F}\left[\tilde{s}^{\alpha(n)}(k, \omega)\right]$,

$\varphi(r, z, t)=\mathscr{F}[\tilde{\varphi}(k, z, \omega)]$.

Then eqs. (2.15) and (3.5) lead to

$\tilde{\varphi}(\boldsymbol{k}, z, \omega)=-\frac{\mathrm{i} \omega}{k} \widetilde{\zeta}(\boldsymbol{k}, t) \mathrm{e}^{k z}$,

with $k=|\boldsymbol{k}|$.

By differentiation of eqs. (2.17) and (3.4) with respect to $t$, and by using eqs. (3.5)-(3.9), one derives

$\xi(k, \omega)=\frac{\mathrm{i} k k_{\alpha} \tilde{s}^{\alpha(1)}(\boldsymbol{k}, \omega)}{\rho g k-p \omega^{2}+\sigma k^{3}-\mathrm{i} \omega k^{3} \chi_{2}}$.

On the other hand, the Fourier transform of eq. (2.12) reads

$$
\begin{aligned}
& \left\langle\tilde{s}^{\alpha(n)}(\boldsymbol{k}, \omega) \tilde{s}^{\beta(n)}\left(\boldsymbol{k}^{\prime}, \omega^{\prime}\right)\right\rangle \\
& =\frac{k_{\mathrm{B}} T \chi_{2}}{8 \pi^{3}} a^{\alpha \beta} \delta\left(\boldsymbol{k}+\boldsymbol{k}^{\prime}\right) \delta\left(\omega+\omega^{\prime}\right) .
\end{aligned}
$$

From eqs. (3.10)-(3.11) one obtains

$$
\begin{aligned}
& \left\langle\tilde{\zeta}(\boldsymbol{k}, \omega) \tilde{\zeta}\left(\boldsymbol{k}^{\prime}, \omega^{\prime}\right)\right\rangle \\
& =\frac{k_{\mathrm{B}} T \chi_{2}}{8 \pi^{2} \rho^{3}} \frac{\delta\left(\boldsymbol{k}+\boldsymbol{k}^{\prime}\right) \delta\left(\omega+\omega^{\prime}\right)}{\left(c^{2}-\omega^{2} / k^{2}\right)^{2}+\left(\omega k \chi_{2} / \rho\right)^{2}},
\end{aligned}
$$

where $c^{2}=g / k+\sigma k / \rho$. By again using Fouricr transformation, one derives for two different points $r$ and $r+\Delta r$

$$
\begin{aligned}
& \langle\zeta(r, t) \zeta(\boldsymbol{r}+\Delta \boldsymbol{r}, t)\rangle \\
& =\frac{k_{\mathrm{B}} T}{2 \pi \sigma} \int_{k_{\min }}^{k_{\max }} \frac{J_{0}(k \Delta r) k \mathrm{~d} k}{k^{2}+\beta^{2}},
\end{aligned}
$$

where $J_{0}$ is a Bessel function, $\Delta r=|\Delta r|, \beta^{2}=\rho g / \sigma$, $k_{\min }$ is a lower cutoff accounting for the finite dimensions of the system and $k_{\text {max }}$ is an upper cutoff accounting for the molecular dimensions. From (3.13) one easily derives the classical result [2$4,16,17]$,

$$
\begin{gathered}
\left\langle\zeta^{2}\right\rangle=\lim _{\Delta r \rightarrow 0}\langle\zeta(r, t) \zeta(r+\Delta r, t)\rangle \\
=\frac{k_{\mathrm{B}} T}{4 \pi \sigma} \ln \left|\frac{1+\left(k_{\max } / \beta\right)^{2}}{1+\left(k_{\min } / \beta\right)^{2}}\right| .
\end{gathered}
$$

In other words, the fluctuation-dissipation approach from section 2 leads to the same result as the approaches of Buff et al. [16] and Grant and Desai [17].

From eq. (3.2), by using a similar derivation, one obtains

$$
\begin{aligned}
\alpha & =\frac{1}{2} \lim _{\Delta r \rightarrow 0}\left\langle\left[\nabla_{\mathrm{II}} \zeta(\boldsymbol{r}, t)\right] \cdot \nabla_{\mathrm{II}} \zeta(\boldsymbol{r}+\Delta \boldsymbol{r}, t)\right\rangle \\
& =\frac{k_{\mathrm{B}} T}{4 \pi \sigma} \int_{0}^{k_{\max }} \frac{k^{3} \mathrm{~d} k}{k^{2}+\beta^{2}} .
\end{aligned}
$$

A lower cutoff is not necessary in eq. (3.15). From eqs. (3.3) and (3.15), one obtains

$f_{\mathrm{cw}}=\frac{\pi}{8} \frac{k_{\mathrm{B}} T}{a^{2}}\left\{1-\left(\frac{\beta a}{\pi}\right)^{2} \ln \left[\left(\frac{\pi}{\beta a}\right)^{2}+1\right]\right\}$,

where

$a=\frac{\pi}{k_{\max }}$

is a parameter of the order of the distance between the centers of two neighboring molecules at the sur- 
face. With $a=7 \AA$, at room temperature, from eq. (3.16) one estimates $f_{\mathrm{cw}}=3.3 \mathrm{mN} / \mathrm{m}$ at $\sigma=30 \mathrm{mN} /$ m (this corresponds to a surface dilation $\alpha=0.11$ ).

\subsection{Thin liquid film on solid substratum}

This case is depicted in fig. 2a. The counterpart of eq. (3.4) reads

$P_{1}=\Pi(\tilde{h})-\rho g \zeta-\left.\rho \frac{\partial \varphi}{\partial t}\right|_{z=0}, \quad P_{\mathrm{II}}=$ const.

where $\tilde{h}=h+\zeta$ is the local instantaneous thickness of the film, $I(\tilde{h})$ is the disjoining pressure, accounting for the excess van der Waals, electrostatic, etc., interactions in the thin film - see, e.g. refs. [5,18]. For $\zeta \ll h$, in linear approximation one can write $\Pi(\tilde{h})=\Pi(h)+\Pi^{\prime}(h) \zeta$. Since at the solid surface,

$\left(\frac{\partial \varphi}{\partial z}\right)_{z=-h}=0$
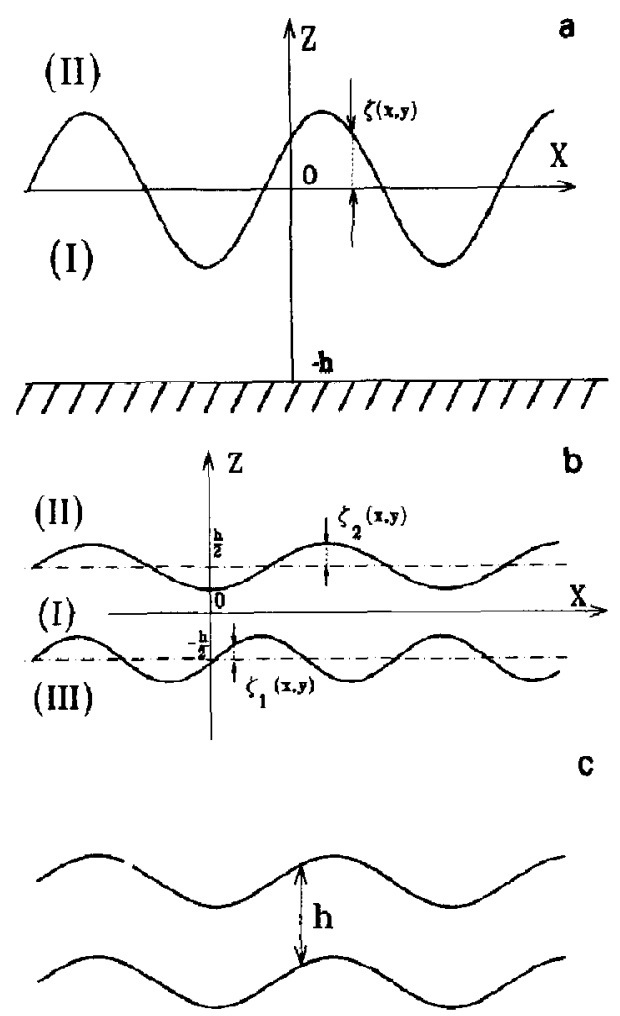

Fig. 2. Fluctuation capillary waves on the surface of a thin liquid film of thickness $h$ : (a) film on solid substrate; (b) free liquid film; (c) bending mode. instead of eq. (3.9) one has

$\tilde{\varphi}(k, z, \omega)=-\frac{i \omega}{k} \bar{\zeta}(k, t) \frac{\cosh [k(z+h)]}{\sinh k h}$.

Then, the counterpart of eq. (3.10) is

$\tilde{\zeta}(\boldsymbol{k}, \omega)=\frac{\mathrm{i} k k_{\alpha} \tilde{S}^{\alpha(n)}(\boldsymbol{k}, \omega)}{G k-\rho \omega^{2} \operatorname{coth} k h+\sigma k^{3}-\mathrm{i} \omega k^{3} \chi_{2}}$,

where

$G=-\Pi^{\prime}+\rho g$.

$\Pi^{\prime}=\left.(\mathrm{d} \Pi / \mathrm{d} h)\right|_{\zeta=0}$ is negative for a stable or metastable thin liquid film - see, e.g. ref. [19]. (Since for such a thin film usually $h<100 \mathrm{~nm}$, as a rule $-\Pi$ ' $\gg g$.)

The analog of eq. (3.16) for a film on a solid substrate is

$$
\begin{aligned}
& f_{\mathrm{cw}}(h)=\frac{\pi}{8} \frac{k_{\mathrm{B}} T}{a^{2}} \\
& \times\left\{1-\left(\frac{\beta_{\mathrm{1}} \mathrm{a}}{\pi}\right)^{2} \ln \left[\left(\frac{\pi}{\beta_{1} a}\right)^{2}+1\right]\right\},
\end{aligned}
$$

with

$\beta_{1}^{2}=\frac{G(h)}{\sigma_{\mathrm{f}}}$.

Here, $\sigma_{\mathrm{f}}$ is the thin film surface tension, which is slightly different from the surface tension $\sigma$ of the bulk liquid because of the disjoining pressure effect - see, e.g. eq. (97) in ref. [18]. For a thick film (large $h), \Pi^{\prime} \rightarrow 0, \beta_{1} \rightarrow \beta$ and eq. (3.23) reduces to eq. (3.16). The cxcess frce energy of a film of thickness $h$, with respect to that of an interface (i.e., infinitely thick film) is

$$
\begin{aligned}
& \Delta f_{\mathrm{cw}}(h)=f_{\mathrm{cw}}(h)-f_{\mathrm{cw}}(\infty) \\
& \quad=-\frac{k_{\mathrm{B}} T}{8 \pi}\left[\beta_{1}^{2}(h) \ln \left(1+\frac{\pi^{2}}{a^{2} \beta_{1}^{2}(h)}\right)\right. \\
& \left.\quad-\beta^{2} \ln \left(1+\frac{\pi^{2}}{a^{2} \beta^{2}}\right)\right] .
\end{aligned}
$$

Since for thin films, $\beta_{1}^{2} \gg \beta^{2}, \Delta f_{\mathrm{cw}}$ is negative. Hence, the damping of the fluctuation capillary waves by the repulsive forces in the film (at equilibrium $\Pi>0$ ) 
results in an effective decrease of the film surface free energy (surface tension).

\subsection{Free thin liquid film}

Let us consider a horizontal thin liquid film (phase I) between two gaseous phases (II and III) - fig. $2 \mathrm{~b}$. It is convenient to choose as the plane $z=0$ the midplane of the film. $\zeta_{1}(x, y)$ and $\zeta_{2}(x, y)$ represent the fluctuation waves on the two film surfaces. Now we have two counterparts of eq. (2.17):

$$
\begin{aligned}
\Delta P_{b} & =\sigma_{f} \Delta_{2} \zeta_{h}+\chi_{2} \Delta_{2}\left(\frac{\partial \zeta_{b}}{\partial t}\right)+s_{b}^{\alpha(n)}{ }_{, \alpha}, \\
b & =1,2,
\end{aligned}
$$

where $\Delta P_{1}=P_{1}-P_{\mathrm{II}}$ and $\Delta P_{2}=P_{\mathrm{III}}-P_{\mathrm{II}}$. The random transverse shear resultants of the two film surfaces, $s_{1}^{\alpha(n)}$ and $s_{2}^{\alpha(n)}$, are not correlated. Then, eq. (2.12) can be generalized as follows:

$$
\begin{aligned}
& \left\langle s_{a}^{\alpha(n)}(\boldsymbol{r}, t) s_{b}^{\alpha(n)}\left(\boldsymbol{r}^{\prime}, t^{\prime}\right)\right\rangle \\
& \quad=2 k_{\mathrm{B}} T \chi_{2} a^{\alpha \beta} \delta\left(\boldsymbol{r}-\boldsymbol{r}^{\prime}\right) \delta\left(t-t^{\prime}\right) \delta_{a b} ; \\
& a, b=1,2 .
\end{aligned}
$$

In addition, one can write

$P_{1}-P_{\mathrm{II}}=\Pi(\tilde{h})-\left.\rho \frac{\partial \varphi}{\partial t}\right|_{z=h / 2}+$ const. ,

$P_{1}-P_{\mathrm{III}}=\Pi(\tilde{h})-\left.\rho \frac{\partial \varphi}{\partial t}\right|_{z=-h / 2}+$ const.

where the gravity effect is neglected for the sake of simplicity. The local instantaneous film thickness is

$\tilde{h}=h+\zeta_{2}-\zeta_{1}$,

where $h$ is the mean film thickness. In accordance with eq. (2.15), the Fourier transform of the velocity potential has the form,

$\tilde{\varphi}(\boldsymbol{k}, z, \omega)=A \mathrm{e}^{-k z}+B \mathrm{e}^{k z}$,

where the constants $A$ and $B$ are to be determined from the boundary conditions,

$\left.\frac{\partial \varphi}{\partial z}\right|_{z=h / 2}=\frac{\partial \zeta_{2}}{\partial t},\left.\frac{\partial \varphi}{\partial z}\right|_{z=-h / 2}=\frac{\partial \zeta_{1}}{\partial t}$.

Instead of eq. (3.21) for a free thin film, one obtains from eqs. (3.26) and (3.28)-(3.32) a set of two equations:

$$
\begin{aligned}
& p \tilde{\zeta}_{1}+q \tilde{\zeta}_{2}=i k_{\alpha} \tilde{s}_{1}^{\alpha(n)}, \\
& q \tilde{\zeta}_{1}+p \tilde{\zeta}_{2}=i k_{\alpha} \tilde{s}_{2}^{\alpha(n)} .
\end{aligned}
$$

Here,

$p=-\Pi^{\prime}+\sigma_{\mathrm{f}} k^{2}-\rho \frac{\omega^{2}}{k} \operatorname{coth} k h-\mathrm{i} \omega k^{2} \chi_{2}$,

$q=\frac{\rho \omega^{2}}{k \sinh k h}+\Pi^{\prime}$

Eqs. (3.33) and (3.34) imply that the fluctuation waves on the two film surfaces are coupled.

We will omit the details of the further calculations, which are similar to those in section 3.2. The final result for the excess free energy of the film surface reads

$\Delta f_{\mathrm{cw}}(h)=-\frac{k_{\mathrm{B}} T}{8 \pi} \beta_{1}^{2}(h) \ln \left(1+\frac{\pi^{2}}{2 a^{2} \beta_{i}^{2}(h)}\right)$,

with $\beta_{1}^{2}=-\Pi^{\prime}(h) / \sigma_{\mathrm{f}}$ (the gravity effect is neglected). The comparison between eq. (3.25) at $\beta \rightarrow 0$ and eq. (3.35) shows that $\left|\Delta f_{\mathrm{cw}}\right|$ is smaller for the surface of the free film at the same $a$ and $\beta_{1}$. This result could he expected, because in the case of free films the repulsive disjoining pressure cannot suppress the so-called "bending mode" [4] taking place at constant film thickness $h$, see fig. $2 \mathrm{c}$. On the other hand, for wetting films (fig. 2a) this mode is entirely suppressed duc to the rigidity of the lower film surface.

\section{Discussion}

The excess free energy of a film surface, $\Delta f$, is simply related to the film contact angle $\theta$ :

$\cos \theta=1+\frac{\Delta f}{\sigma}$

see, e.g. eq. (109) in ref. [18].

In the case of comparatively thicker free films, stabilized by electric double layer repulsion, $\beta_{1}^{2}=$ $-\Pi^{\prime} / \sigma \approx 10^{9} \mathrm{~cm}^{-2}[20]$. Then, with $a=7 \AA$, from eq. (3.35) one estimates $\Delta f_{\mathrm{cw}} \approx-2.3 \times 10^{-5} \mathrm{mN} / \mathrm{m}$. This value is too small to affect the contact angle, 
whose value in this case is of the order of $1^{\circ}$ [21].

In contrast with the forementioned case of soft repulsion, $\beta_{1}^{2}$ can be very large in the case of hard repulsion. The latter is observed with the so-called Newton black films stabilized with ionic surfactants [19] or with the films sterically stabilized with nonionic surfactants [22]. A typical disjoining pressure isotherm is shown in fig. 3 . The maximum possible value of $\Delta f_{\mathrm{cw}}\left(\right.$ at $\left.\beta_{1} \rightarrow \infty\right)$ is

$\Delta f_{c w}^{\max }=-\frac{\pi k_{\mathrm{R}} T}{16 a^{2}}$,

for free films. At $a=7 \AA$ and room temperature, one obtains $\Delta f_{\mathrm{cw}}^{\max }=-1.6 \mathrm{mN} / \mathrm{m}$. With $\sigma=32 \mathrm{mN} / \mathrm{m}$, from eq. (4.1) one calculates $\theta=18.5^{\circ}$. $\theta$-values of about $8-9^{\circ}$ were measured experimentally with anionic surfactant [23]. Hence, in reality $\left|\Pi^{\prime}\right|$ can be very large, but still less than infinity. Of course, the attractive van der Waals forces and the ion correlations [24] also contribute to the value of $\theta$.

With nonionic surfactants, Lobo et al. [22] mea-

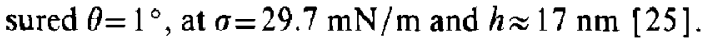
This value of $\theta$ corresponds to $\Delta f=-4.52 \times 10^{-3}$ $\mathrm{mN} / \mathrm{m}$, whereas the contribution of the van der Waals forces is $\Delta f_{\mathrm{vw}}=-2.35 \times 10^{-3} \mathrm{mN} / \mathrm{m}\left(\Delta f_{\mathrm{vw}}\right.$ is calculated by numerical integration of the expression for $\Pi_{\mathrm{vw}}(h)$ provided in ref. [26]). The difference can be attributed to the capillary wave effect: $\Delta f_{\mathrm{cw}}=-2.17 \times 10^{-3} \mathrm{mN} / \mathrm{m}$. The latter value corresponds to $\Pi^{\prime}=-4.5 \times 10^{13} \mathrm{~N} / \mathrm{m}^{3}$. This value seems to be reasonable in view of the hard osmotic repulsion between the interpenetrating polyoxiethylene layers of the two film surfaces.

It should also be noted that in accordance with the thermodynamics of thin films [18], the fluctuation capillary wave effect gives rise to a contribution to

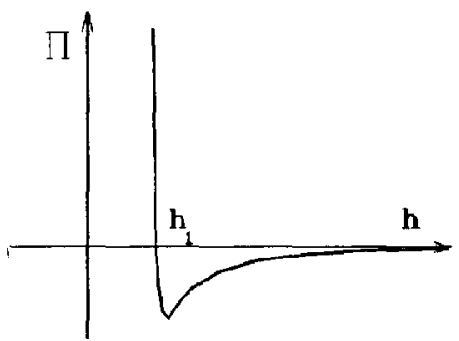

Fig. 3. Typical dependence of the disjoining pressure $\Pi$ on the film thickness $h$ exhibiting hard repulsion at $h \approx h_{1}$. the disjoining pressure: $\Pi_{\mathrm{cw}}=-2\left(\partial \Delta f_{\mathrm{cw}} / \partial h\right)$.

In conclusion, the suppression of fluctuation capillary waves by the repulsive forces in the thin films can give a considerable contribution to the value of the film contact angle, especially in the case of hard repulsion. This result was obtained by applying the fluctuation-dissipation approach developed in section 2. Of course, the latter approach can also be applied to many other capillary wave problems.

\section{Acknowledgement}

This work was financially supported by the Bulgarian Ministry of Science and Higher Education.

\section{References}

[1] L. Mandelstan, Amn. Plıysik 41 (1913) 609.

[2] J.S. Rowlinson and B. Widom, Molecular theory of capillarity (Clarendon Press, Oxford, 1982).

[3] R.C. Desai and M. Grant, in: Fluid interfacial phenomena, ed. C.A. Croxton (Wiley, New York, 1986) p. 135.

[4] J.G.H. Joosten, in: Thin liquid films, ed. I.B. Ivanov (Dekker, New York, 1988 ) p. 569.

[5] I.B. Ivanov and P.A. Kralchevsky, in: Thin liquid films, ed. I.B. Ivanov (Dekker, New York, 1988) p. 49.

[6] L.D. Landau and E.M. Lifshitz, Fluid mechanics (Pergamon Press, Oxford, 1984).

[7] E.M. Lifshitz and L.P. Pitaevsky, Theoretical physics, Vol. 9. Statistical physics 2 (Nauka, Moskow, 1978) (in Russian).

[8] P.M. Naghdi, in: Handhuch der Physik, Vol. 6a/2, ed. S Flugge (Springer, Berlin, 1972) p. 425.

[9] Y.S. Podstrigach and Y.Z. Povstenko, Introduction to mechanics of the surface phenomena in deformable solids (Naukova Dumka, Kiev, 1985) (in Russian).

[10] E.A. Evans and R. Skalak, Mechanics and thermodynamics of biomembrancs, Part 1 (CRC Prcss, Boca Raton, 1979).

[11] A.J. McConnel, Application of tensor analysis (Dover, New York, 1957).

[12] J.D. Eliassen, Ph.D. Thesis, University of Minnesota, Minneapolis (1963) (University Microfilms, Ann Arbour, Michigan, 1983).

[13] P.A. Kralchevsky, J. Colloid Interface Sci. 137 (1990) 217

[14] T.D. Gurkov and P.A. Kralchersky, Colloids Surf. 47 (1990) 45 .

[15] L.E. Scriven, Chem. Eng. Sci. 12 (1960) 98

[16] F.P. Buff, R.A. Lovett and F.H. Stillinger, Phys. Rev. Letters 15 (1965) 621 .

[17] M. Grant and R.C. Desai, Phys. Rev. A 27 (1983) 2577 ,

[18] J.A. de Feijter, in: Thin liquid films, ed. I.B. Ivanov (Dekker, New York, 1988) p. 1. 
[19] P.M. Kruglyakov, in: Thin liquid films, ed. I.B. Ivanov (Dekker, New York, 1988) p. 767.

[20] D. Exerowa, T. Kolarov and Khr. Khristov, Colloids Surf. 22 (1987) 171 .

[21] F. Huisman and K.J. Mysels, J. Phys. Chem. 73 (1969) 489.

[22] L.A. Lobo, A.D. Nikolov, A.S. Dimitrov, P.A. Kralchevsky and D.T. Wasan, Langmuir 6 (1990) 995.
[23] P.A. Kralchevsky, A.D. Nikolov and I.B. Ivanov, J. Colloid Interface Sci. 112 (1986) 132

[24] R. Kjelander and S. Marčelja, Chem. Phys. Letters 112 (1984) 49.

[25] A.D. Nikolov, D.T. Wasan, N.D. Denkov, P.A. Kralchevsky and I.B. Ivanov, Progr. Colloid Polym. Sci. 82 (1990) 87.

[26] Chr.St. Vassilieff and I.B. Ivanov, Colloid Polymer Sci. 254 (1976) 431 